\title{
Strategies and support for Black, Indigenous, and people of colour in ecology and evolutionary biology
}

We know that it can be tough to be a person of colour working in ecology and evolution. Here is what has worked for us.

\author{
Michelle Tseng, Rana W. El-Sabaawi, Michael B. Kantar, Jelena H. Pantel,
} Diane S. Srivastava and Jessica L. Ware

T he field of ecology and evolutionary biology (EEB) has strikingly low racial diversity, with Indigenous and Black peoples particularly underrepresented ${ }^{1-5}$. For example, between 2014 and 2018, only $1 \%$ of EEB PhD graduates in the United States were Black or African American and $0.2 \%$ were American Indian or Alaska Native. This is in contrast to $14 \%$ and $2 \%$ of Americans identifying as Black/African American and American Indian/Alaska Native, respectively.

The reasons for the overwhelming 'whiteness' in EEB are too lengthy to list here but they include barriers and discrimination at every stage of the academic ladder (see visual representation in Fig. 1). Challenges to inclusion for undergraduates included fewer same-race role models and a lower overall sense of belonging ${ }^{3}$. In a study where researchers sent fabricated CVs of evolutionary biology postdoctoral candidates to potential supervisors, Black and Latinx candidates were ranked less competent than white candidates despite identical $\mathrm{CVs}^{6}$. Certain journals in EEB are more likely to reject papers from particular geographic regions, and in academia in general, contributions by minority groups are devalued and discounted compared to equivalent work done by majority groups ${ }^{8}$.

EEB tackles some of the most complicated problems facing the world today - the collapse of global biodiversity, widespread antibiotic resistance, invasive species moving into every nook and cranny of the planet, just to name a few. Fifty years of research on heterogeneity of organizations and populations has repeatedly shown that diverse working groups are more productive and have greater problem-solving ability ${ }^{9,10}$. For our field to succeed we need to dismantle the barriers faced by Black, Indigenous, and people of colour (BIPOC), stem the loss of diverse talent, and elevate support for BIPOC working in EEB today.

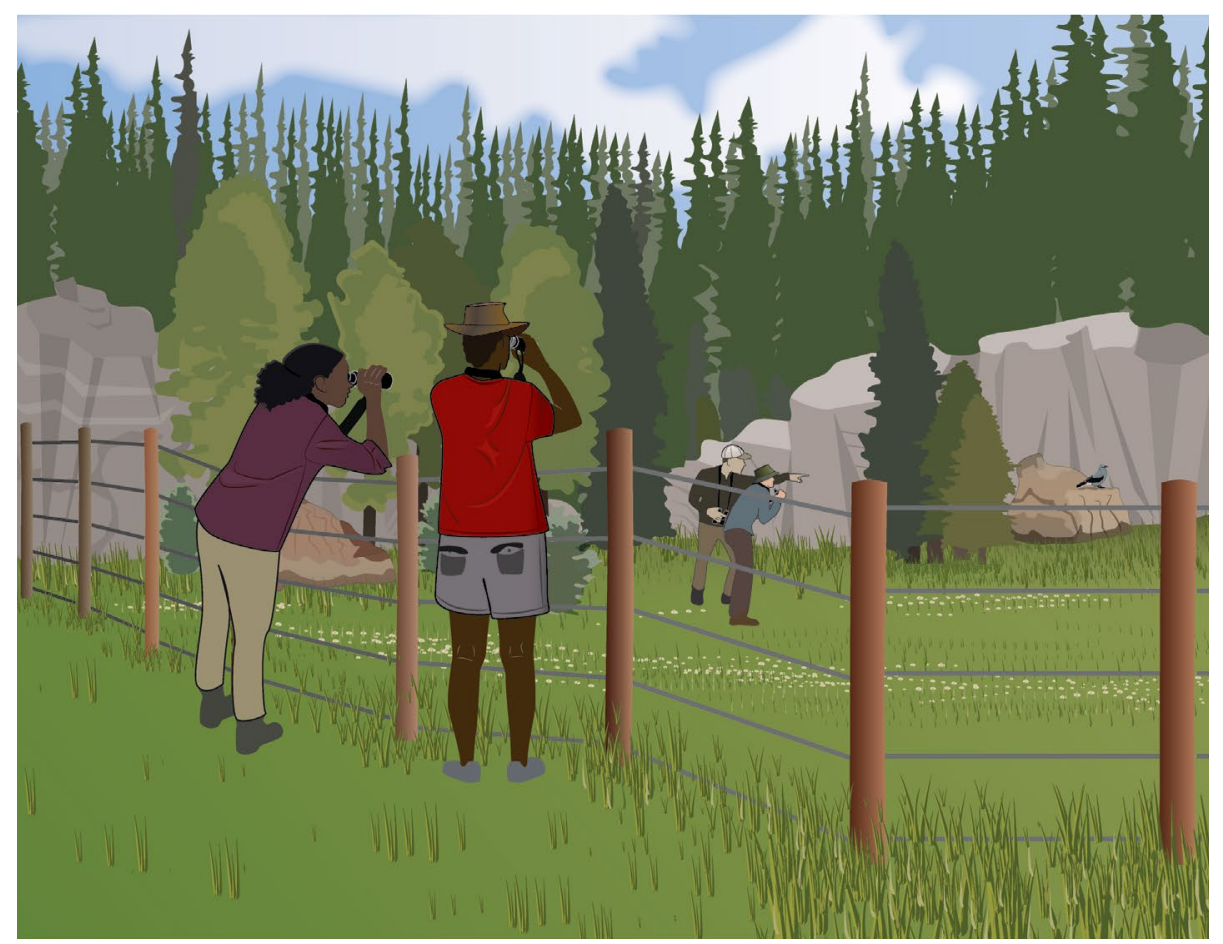

Fig. 1 | Barriers. See text for details. Credit: illustration by Sylvia Heredia

Those of us here identify as BIPOC working in $\mathrm{EEB}$, are at different stages of our academic careers, and we have held positions in and out of academia. In the aftermath of the senseless killings of Ahmaud Arbery, Breonna Taylor, George Floyd, and too many others to name, BIPOC in EEB have been under enormous pressure to help non-BIPOC educate themselves, to write statements of support, or to guide their research groups in discussions of racism. The focus, ironically, has been on how to help the majority. Here we reach out to BIPOC in EEB in academia. We have compiled some strategies (Fig. 2) that we hope will help fellow BIPOC with the many barriers that lie ahead. Like other BIPOC researchers in EEB, we each come from different cultural and socio-economic backgrounds, we exist along different intersectional axes, and we have faced varying degrees of racism. We recognize and respect that the lived experiences of BIPOC in EEB are highly variable, and that other BIPOC researchers may not agree with our strategies.

\section{Take care of your mental health}

First and foremost, take care of your mental health, nourish your soul, and be kind to yourself. Being the only BIPOC in a department, a field station, a meeting, 


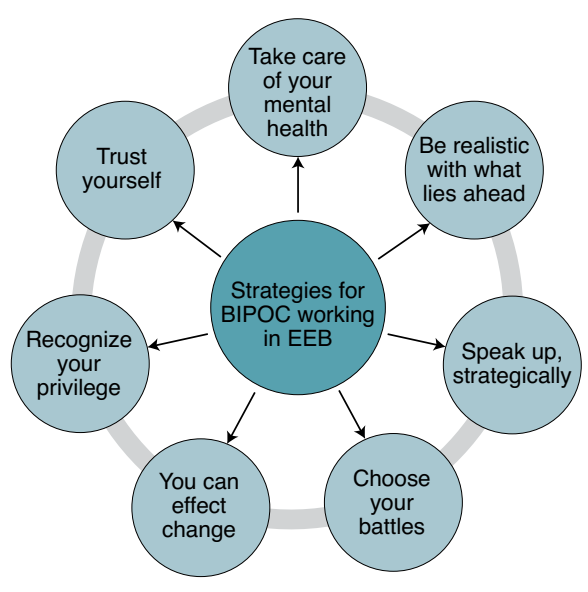

Fig. 2 | Summary of strategies for Black, Indigenous, and people of colour working in ecology and evolutionary biology. See text for details.

day in and day out is exhausting and corrosive. Being immersed in a homogeneous culture of the majority can make you doubt yourself and feel lonely. Your colleagues will not understand things that routinely happen to you (for example, police interrogations at field sites, being overlooked at conferences, assumptions that you hold a less-skilled position, overly personal questions about your appearance). For BIPOC, self-care is a radical act of self-preservation. It can take many forms, such as therapy or a hobby, anything that feels restorative and nourishing. Having a supportive network within and outside academia is an antidote for loneliness, but so is connecting with BIPOC from all walks of life. Anything that makes you feel seen or understood in a culture that does not will help remind you that the EEB world is not representative of the world at large.

\section{Be realistic with what lies ahead}

The lack of diversity in EEB means that there are not enough people who understand how institutional racism in academia is going to disproportionately affect you in graduate school, when science budgets are slashed, when you contact people for postdoc placements, and when you go on the job market. Try to get advice from other BIPOC to find job opportunities with mentors who can truly understand what you are facing, as your white colleagues might not understand the power dynamics at play for BIPOC in labs and research groups. The references we included here highlight some of the battles that lie ahead, but these do not include dealing with people who have never had to accept 'no' for an answer, seeing your grants get rejected when white colleagues with seemingly equivalent scores are funded, and the emotional turmoil associated with constantly reminding yourself that you do belong in EEB, your papers are ground-breaking and innovative, and you do deserve that grant, that salary, and that raise - and when you achieve these, it is not just out of tokenism. Seek out those colleagues who can help you chart your own successful path despite these obstacles, and avoid those who do not understand that it is not you who needs to try harder.

\section{Speak up, strategically}

The barriers experienced by women of colour in particular are unique, and are not just a hybrid of the difficulties faced as a woman and as a person of colour. In majority-white cultures, women of colour have often ended up in some of the poorest paid and most precarious jobs, especially those involving service to others. This is often replicated within universities and leads to a specific type of implicit bias, such as assumptions that you are not the leader of a research team but more a junior member. One effective defence is to be quick to introduce yourself in the way you want to be known, but this can also be draining and can come with risks.

Speaking up only works if you are being heard. BIPOC know that their voices are listened to and perceived differently than white voices. You can still watch and learn some strategies from those with voices that are heard more easily than yours. While biases persist and you are not truly allowed to speak in the same register, you can still employ some of the same arguments to support your ideas. If you feel you cannot speak out, perhaps seek an ally on your team to do so. Insist that your contributions be recognized, even if this means uncomfortable conversations about things like authorship. Our experience is that this will sometimes open your colleague's eyes, and result in an apology and ultimately a better working relationship.

If you feel like you are swimming against the tide, you probably are. Pursue opportunities that allow you to work with people that are not only great scientists, but who respect you as a person and give you the space that you may be denied elsewhere. When you have made it to a leadership position, focus on creating a team culture of inclusiveness, communication, and thoughtfulness.

Choose your battles and pace yourself Most BIPOC in science, technology, engineering and mathematics (STEM) feel a deep responsibility to make academia more inclusive. Institutions can change via top-down or bottom-up action. The former includes difficult fighting and grappling with big systems and their administrators. The latter involves building supportive networks, mentoring BIPOC students, and ensuring that day-to-day operations are as inclusive as possible. Remember that you can choose your battles, and that it is unwise to fight all of the battles all of the time. Your choice may depend on where you are in your career. The bottom-up approach might be more suitable in pre-tenure or mid-career positions, while the top-down battles can be fought in post-tenure or late-career roles.

BIPOC scientists, and those facing intersectional barriers, often are called upon for countless hours of service, much of which is not easily quantifiable in tenure packets and on CVs. It can be important to set boundaries for yourself, to prevent burnout. If your work as a 'role model' prevents you from reaching your academic goals, it can be helpful to prioritize yourself and your time, while also advocating for codified policies at your institution to support such mentoring efforts. Remember that just because you are a BIPOC voice, it does not mean you have to be the face of all BIPOC issues for your department, centre or institution. Repeatedly fielding requests to address racism is emotionally and physically exhausting. Pace yourself, and do not be afraid to say no.

\section{Ask questions}

Unfortunately, many institutions believe that the solution to the diversity problem is only to hire people from under-represented groups or people of colour, but without making important systematic changes to help them thrive. To these institutions diversity is simply a numbers game, rather than a truly inclusive system. Before accepting a position, take a closer look. Ask specifically about inclusion policies 
or programmes, and ask BIPOC faculty whether the institution has demonstrated that it is a supportive environment. Joining a lab or university that is not inclusive will cost you more energy and can slow down your career progress. You are more powerful than you realize to decide what kind of group you want to work in, as universities increasingly understand that retention problems reflect poorly on them.

As BIPOC researchers, our presence on panels and committees is a powerful thing. It makes us visible to our community and to current and future students. For organizers, it unfortunately also can be used as a legitimizing factor, a tidy check mark for 'diversity', regardless of whether the event is actually supportive of people from under-represented groups. You can flip this tokenism around and use your presence on a committee as leverage. Before you accept membership, ask questions about inclusive policies, and if you are not satisfied with the answer, then say no.

\section{You can inspire and effect change}

If you are any type of BIPOC, your presence alone in academia will inspire BIPOC early-career researchers. Be mindful with this unexpected influence. Welcome students into your office even when you are under deadlines, as these few minutes may sway their career path. Tell them about the delight and joy you find in science, as these positives will help make the barriers seem small by comparison.

Often institutions, granting agencies, editorial boards, and scientific societies can seem like exclusionary fortresses. To break down barriers takes time, and long-term societal change. At the faculty level, we can start by making clear what we expect from our students and postdocs as we work with them toward our common goal of breaking down systemic racism in academia. When we serve on committees, we can point out bias, and advocate for policies that promote equity. A warning, however, is that in doing so we may risk being marginalized and this is of particular concern before tenure.

As teachers and instructors, we have the opportunity as BIPOC in science to discuss ecology and evolution in the context of historical racism (for example, the complicity of evolutionary biologists in eugenics, or ecological impacts of colonialism), and modern inequality (for example, discounting of Indigenous knowledge by the academy) in our classrooms. We can have integrative materials that teach EEB fundamentals while comparing important findings to the timeline of social movements such as civil rights, abolition, or self-governance. We can include people that look like us in our course materials, on posters, and in talks at meetings.

\section{Recognize your own privilege and trust yourself}

Finally, while academia sometimes feels like it is all encompassing, when you are working and communicating with the non-academic community, and especially with other under-represented communities, you may not be viewed as a minority group, but rather as a member of the ivory tower. Recognize this privilege in order to move these interactions forward, and to break barriers between academia and the general public. Most importantly, be aware of your own biases and continue to educate yourself to try to avoid the mistakes of the past. Think carefully about the ways that your community can be both the victim of discrimination and still perpetuate discrimination against other BIPOC.

Do the ecology and evolutionary biology that you love, and that drives you to pursue excellence. The unfortunate truth is that widespread bias against BIPOC scholars means that BIPOC need to run twice as fast as non-BIPOC just to keep up. Your passion for EEB and your instincts for survival will help you balance the daily struggles. Trust yourself. You have skills that colleagues who have not had to fight additional racial roadblocks do not have. EEB needs people like you, and the field is beginning to see this.

Michelle Tseng (iD) 1,2凶, Rana W. El-Sabaawi ${ }^{3}$, Michael B. Kantar (D), Jelena H. Pantel ${ }^{5}$, Diane S. Srivastava (D) ${ }^{1}$ and Jessica L. Ware (iD) 6

${ }^{1}$ Department of Zoology and Biodiversity Research Centre, University of British Columbia, Vancouver, British Columbia, Canada. ${ }^{2}$ Dept of Botany, University of British Columbia, Vancouver, British Columbia, Canada. ${ }^{3}$ Department of Biology, University of Victoria, Victoria, British Columbia, Canada. ${ }^{4}$ Department of Tropical Plant and Soil Sciences, University of Hawaii at Manoa, Honolulu, HI, USA. ${ }^{5}$ Department of Computer Science, Mathematics, and Environmental Science, The American University of Paris, Paris, France. ${ }^{6}$ Invertebrate Zoology, American Museum of Natural History, New York, NY, USA.

$\varpi_{e-m a i l: m t s e n g @ z o o l o g y . u b c . c a}$

Published online: 7 July 2020

https://doi.org/10.1038/s41559-020-1252-0

\section{References}

1. Qian, W., Lachapelle, F., Fuller, S., Corrigall-Brown, C. \& Srivastava, D. S. Preprint at bioRxiv https://doi org/10.1101/2020.05.12.092247 (2020).

2. Graves Jr, J. L. Evol. Educ. Outreach 12, 18 (2019).

3. O'Brien, L. T., Bart, H. L. \& Garcia, D. M. Soc. Psychol. Educ. 23, 449-477 (2020).

4. Brown, K. H., McHatton, P. A. \& Scott, M. F. in Faculty of Color Navigating Higher Education (eds Brown, K. H., McHatton, P. A. \& Scott, M. F. T.) Ch. 1 (Rowman and Littlefield, 2017).

5. Wanelik, K. M., Griffin, J. S., Head, M. L., Ingleby, F. C. \& Lewis, Z. Ecol. Evol. https://doi.org/10.1002/ece3.6423 (2020).

6. Eaton, A. A., Saunders, J. F., Jacobson, R. K. \& West, K. Sex. Roles 82, 127-141 (2020).

7. Edwards, H. A., Schroeder, J. \& Dugdale, H. L. PLoS ONE 14, e0201725 (2018).

8. Hofstra, B. et al. Proc. Natl Acad. Sci. USA 117, 9284-9291 (2020). 9. Gomez, L. E. \& Bernet, P. J. Natl Med. Assoc. 111, 383-392 (2019). 10. Cooke, A. \& Kemeny, T. Res. Policy 46, 1175-1185 (2017).

Competing interests

The authors declare no competing interests. 